\title{
FORUM
}

\section{Rate and Ratio in Radar Collision Avoidance}

\author{
J. Vendrell
}

Most authorities are agreed that detailed plotting is an essential part of a ship's radar collision-avoidance system, and teaching on the subject includes the calm intellectual detachment of the radar simulator; with First and Second Officers respectively to plot the data and evaluate the developing situation, decide upon the appropriate action and initiate the conn. In conflict with this ideal executive bridge manning concept is the increasing tendency towards automation and resultant crew reduction, to the point where in an increasing number of vessels (in particular the ever increasing number of specialized supply vessels in the offshore oil industry) spare capacity no longer exists to permit the separation of bridge functions such as lookout (Rule 29), radar observer, radar plotter and anti-collision conning. The dictates of low manning, long hours and arduous and often dangerous work on low freeboard, heaving sea-swept decks, all combine in such vessels to restrict bridge manning to a single navigating officer-as a rule on a six-hour shift-overseeing the gyro-steering, reading out and plotting from the position fixing system, operating the radios, keeping a weather eye on the various bridge-mounted engine gauges, observing the radar and, when necessary, overriding the gyro steering to manouvre the ship manually in any collision-potential situation. Radar plotting is seldom undertaken in this class of vessel, there being a limit even to the accomplishments of the one-man-band.

To the navigator in such vessels I would offer the principle of using 'rate and ratio' to evaluate the generation of collision risk and the determination of avoiding action, where this becomes necessary in a radar encounter. Some elementary arithmetic is involved which can be adequately handled on the least expensive and simplest of the modern miniature hand-held electronic calculators. Beyond that, and to eliminate confusion, I would recommend maintaining a radar log in the form shown in Table I, with times appropriate to each target kept together even if this results in successive entries on the page not being in strict chronological sequence. By 'rate' I mean the relative velocity by which a radar target is seen to be closing (as distinct from opening) its range from own ship. By 'ratio' I mean the ratio found to exist between own ship's and target's contributions to 'rate'.

Obviously a target whose bearing alters appreciably in relation to time is not a collision risk and may, circumspectly, be disregarded. On the other hand targets whose bearings remain steady or nearly steady while closing are high collision risks and, specifically because of their steady bearing, can be assessed for closing rate simply by noting the time of their transit between two successive radar range rings. On radar ranges between ten and fifteen miles most radars seem to be graduated with range rings spaced two miles apart, so the electronic calculator can be used to calculate rate by solving: Rate $=120 /$ (minutes between rings). Measured rate I call 'gross rate' and designate $R$. It is composed of ele- 
No. I

FORUM

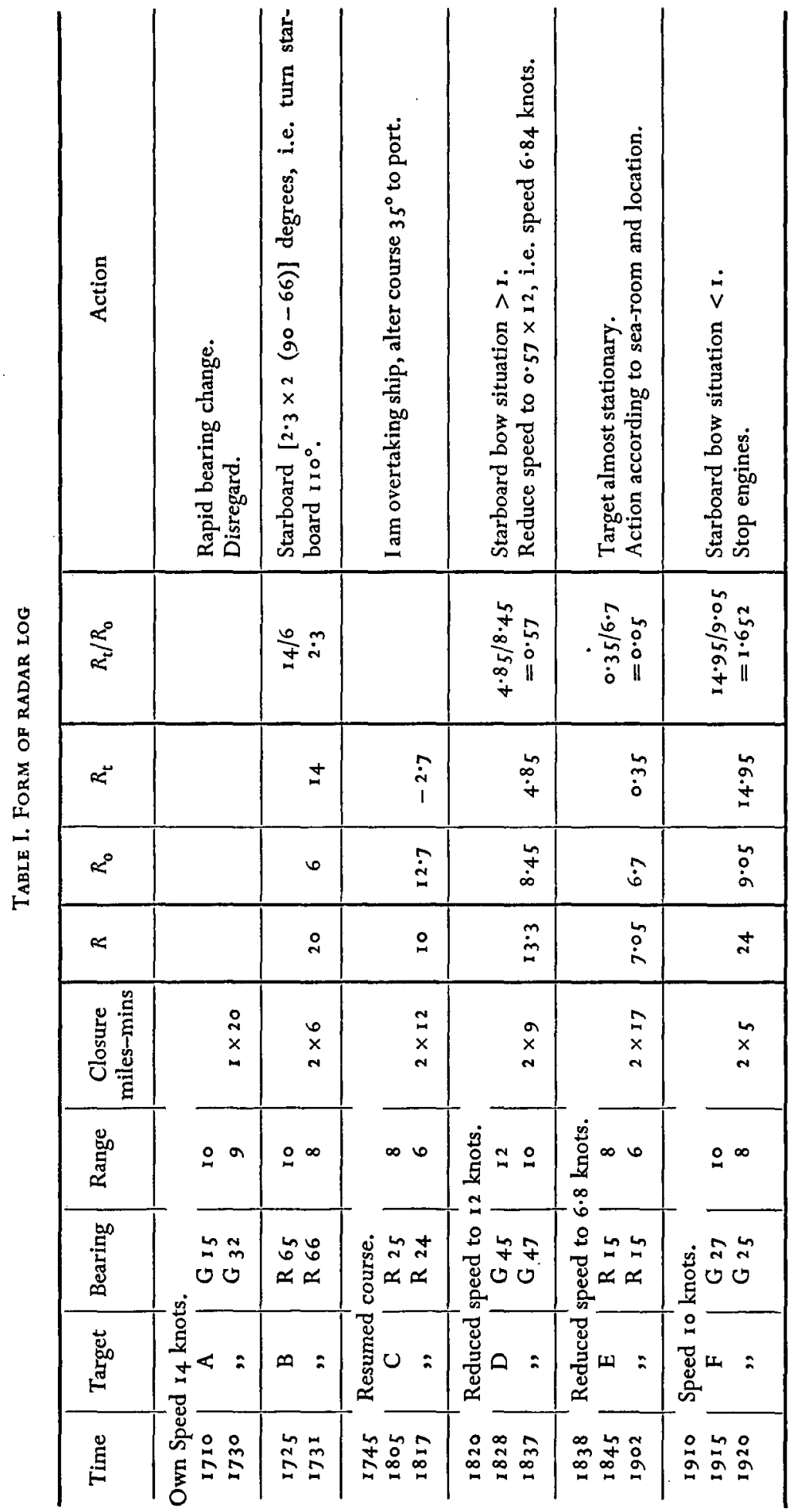


ments contributed both by own ship and target; I call these respectively $R_{\mathrm{o}}$ (own ship's component) and $R_{t}$ (target's component) and express their relationship: $R=R_{\mathrm{o}}+R_{\mathrm{t}}$ and $R-R_{\mathrm{o}}=R_{\mathrm{t}}$.

Now $R_{0}$ is derived from own ship's speed $(V)$ multiplied by the cosine of target's angle on the bow $(\theta)$, so that $R_{\mathrm{o}}=V \cos \theta$. Consider a stationary object positioned right ahead of own ship. It is obvious that its closing rate as seen on radar would be equal to own ship's speed. An object stationary on the beam would however be seen on radar to be neither opening nor closing its range, or in other words to have a zero rate. One of the more sophisticated small calculators having trig. functions could be used to compute $R_{\mathrm{o}}$ or, to keep within the capability of the less expensive instruments, a diagram such as that in Fig. I can be kept handy, or even inside the radar log. Anticipating some criticism of the high speeds shown in this figure (vis-a-vis Rule I6) I would explain that I compiled it some years ago for use in high speed search and rescue craft in which, when life was at risk, Lord Nelson's example at Copenhagen was kept in mind. The tabulation is submitted as an example and the values are shown for port bow, but of course hold good for starboard bow. Incidentally, values are positive for targets forward of the beam and negative for those abaft the beam. The latter however are not considered further in this paper.

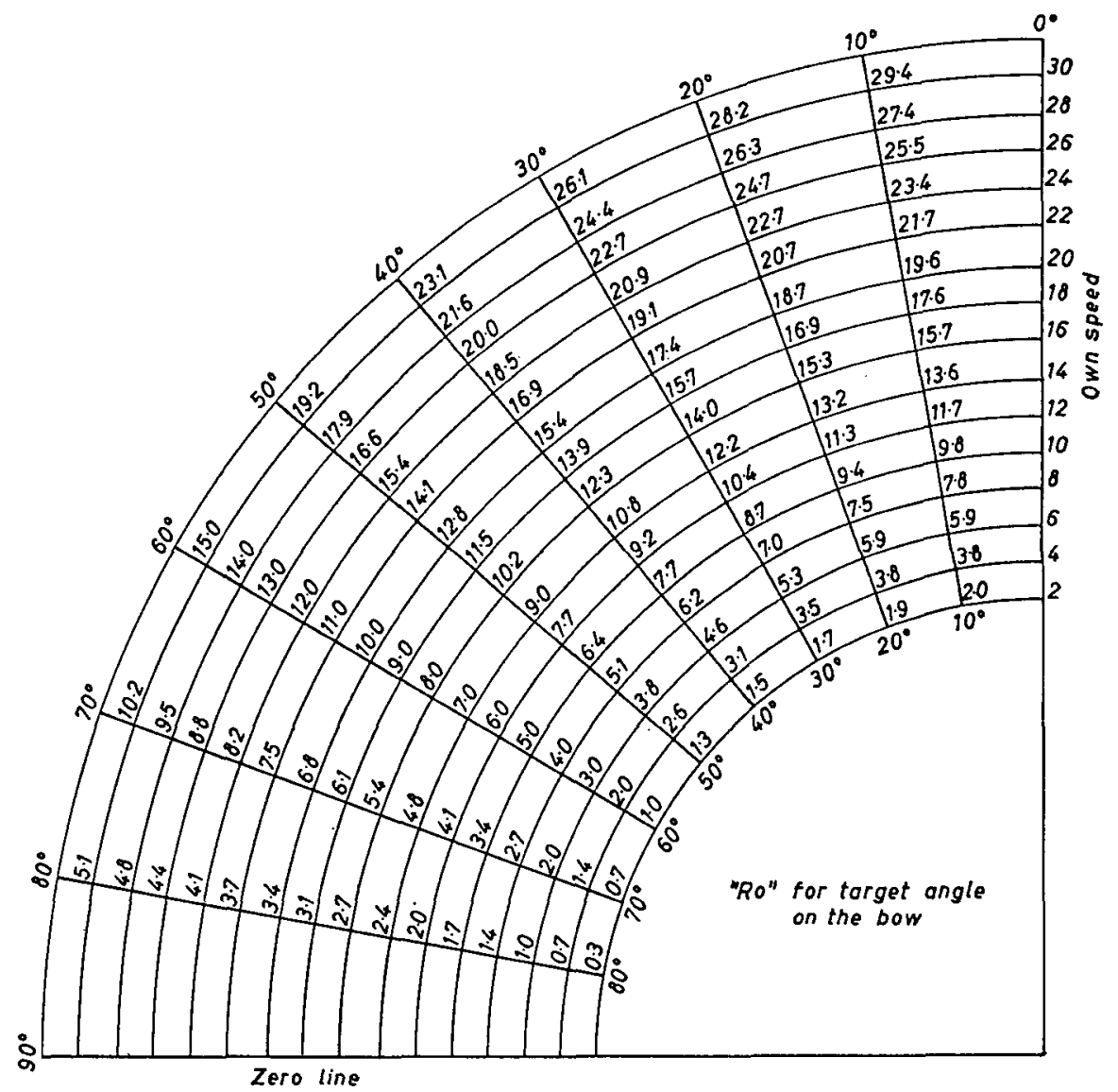

FIG. I 
From the equation $R_{t}=R-R_{0}$, the target's component of the closing rate is obtained either mentally or by calculator. While the parameters governing own ship's component are known exactly those for $R_{\mathrm{t}}$ are not, and an infinite number of permutations of target course and speed exist to produce a given value for $R_{t}$. Nevertheless, the value of $R_{t}$ together with its algebraic sign yields valuable information and the ratio $R_{\mathrm{t}} / R_{\mathrm{o}}$ (from the calculator) provides a factor to be used in deciding the degree of avoiding action necessary to eliminate collision risk. The following is the basic concept:

If $R_{\mathrm{t}}$ is negative: I am 'overtaking' ship and must alter course to put the target at least $10^{\circ}$ on the opposite bow.

If $R_{t}$ is zero or nearly zero: Not forgetting the prevailing tidal stream the target is stationary; action is according to chart location and sea room.

If $R_{t}$ is positive: Target is inclined towards me and I must now assess and operate the ratio $R_{t} / R_{\mathbf{o}}$.

(i) If $R_{t} / R_{\mathrm{o}}$ is 1 or greater than 1 : Target's component being equal to or greater than my own, assuming target will take no avoiding action, my own action must be sufficient to eliminate or negate the gross rate.

Target to starboard: stop engines.

Target to port turn to starboard $\left(2 x \times R_{\mathrm{t}} / R_{\mathrm{o}}\right)$ degrees.

(ii) If $R_{\mathrm{t}} / R_{\mathrm{o}}$ is less than 1 : My own ship is generating most of the collision risk so that a proportionally smaller action on my part will eliminate the risk.

Target to starboard: reduce speed to $R_{\mathrm{t}} / R_{\mathrm{o}} \times$ present speed.

Target to port: turn to starboard $2 x \times R_{\mathrm{t}} / R_{\mathrm{o}}$ degrees.

In defining $x$ and $2 x$ I am quoting the turn advocated by the French Institute of Navigation (London meeting 1957), where $x$ is defined as the target's angle forward of the port beam and $2 x$ a turn to starboard of twice that angle. In plain language: turn to starboard until the target comes abeam to port, then continue the turn by the same amount again. A $2 x$ turn was at one time thought to be sufficient but a plot out of the example of target ' $B$ ' in Table I will show that this is not so. I prefer to define a $2 x$ turn as a 'standard' turn to be adjusted by the ratio $R_{t} / R_{0}$.

In conclusion, I would stress again that the measurement of 'rate' is in practice most accurate when collision risk is greatest, that is to say when the target on a relative motion display is seen to be closing on a radius cutting across the range rings at right angles. In practice I have found the principles described to work well enough for changes in bearing up to five degrees in two miles of closing range. Beyond that the accuracy in the measurement of rate falls away rapidly, but so too does the collision risk.

\section{Education and the Sea}

\section{W. J. Fifield}

Professor M'Pherson's paper on 'Education and the Sea'1 does somewhat less than justice to the non-university sector of the British educational system; his Appendix $A$ lists only two university undergraduate courses in nautical/maritime 